\title{
Nanoparticle Flow in Polymer Grafted Channels
}

Sean Burgess ${ }^{l}$, Kolattukudy P. Santo ${ }^{l}$, Yefim Brun ${ }^{2}$, Alexander V. Neimark ${ }^{1 *}$

${ }^{l}$ Department of Chemical and Biochemical Engineering, Rutgers, The State University of New Jersey, 98 Brett Road, Piscataway, New Jersey 08854, USA

${ }^{2}$ DuPont Central Research and Development, Wilmington, Delaware 19803, United States

\section{Corresponding Author}

"E-mail: aneimark@rutgers.edu

\section{Supplementary Information.}

\section{Dissipative Particle Dynamics Setup}

Dissipative particle dynamics (DPD) ${ }^{1-2}$ is employed to study hydrodynamic flow of a fluid in a polymer brush coated channel. DPD is a coarse grain simulation technique which uses Newton's equation of motion to monitor the dynamics of equilibrium conditions of a system. It is frequently used to study fluid dynamics and polymer interactions. ${ }^{3-4}$ In DPD, bead positions evolve over time according to (1)

$$
d \mathbf{r}_{\mathbf{i}} / d t=\mathbf{v}_{i} ; \quad d \mathbf{v}_{i} / d t=\mathbf{f}_{i}
$$

Forces between the beads are calculated using (2)

$$
\mathbf{f}_{i}=\sum_{j \neq i}\left(\mathbf{F}_{i j}^{(C)}+\mathbf{F}_{i j}^{(B)}+\mathbf{F}_{i j}^{(D)}+\mathbf{F}_{i j}^{(R)}\right)
$$

Where $\mathbf{F}_{\mathrm{ij}}^{(C)}$ is the 'conservative' interaction force between two beads, $\mathbf{F}_{i j}^{(B)}$ the bond force, $\mathbf{F}_{i j}^{(D)}$ an imposed drag force, and $\mathbf{F}_{i j}^{(R)}$ and imposed random force. One of the main advantages of DPD is its increases computational efficiency when compared with coarse-grained molecular dynamics (MD). This is largely due to the 'soft' bead force which allows for larger timesteps since bead overlap does not carry a high energy penalty as it does in MD,

$$
\mathbf{F}_{i j}^{C}= \begin{cases}-a_{i j}\left(1-\left|r_{i j}\right| / R_{c}\right) & \text { if }\left|r_{i j}\right| \leq R_{c} \\ 0 & \text { if }\left|r_{i j}\right|>R_{c}\end{cases}
$$

Where $a_{i j}$ is the maximum interactional potential felt between two beads $i$ and $j, r_{i j}$ is the magnitude of their distance, and $R_{c}$ is the effective bead diameter equaled the cutoff of the interbead interaction potential. The bond force between two beads is harmonic and is described in (4)

$$
\mathbf{F}_{l j}^{(\mathrm{B})}=-K_{l}^{(\mathrm{B})}\left(r_{i j}-r_{\mathrm{e}}\right) \hat{r}_{\mathrm{ij}}
$$


Where $K_{l}^{(\mathrm{B})}$ is the spring constant, and $r_{\mathrm{e}}$ is the equilibrium spring distance. The random and drag forces work in conjunction similarly to a Langevin thermostat

$$
\begin{array}{r}
\mathbf{F}_{i j}^{(\mathrm{R})}=\sigma w^{(\mathrm{R})}\left(r_{i j}\right) \theta_{i j}(t) \hat{r}_{i j} ; \quad \mathbf{F}_{\mathrm{ij}}^{(\mathrm{D})}=-\gamma w^{(\mathrm{D})}\left(r_{i j}\right)\left(\hat{r}_{i j} \cdot v_{i j}\right) \hat{r}_{i j} \\
w^{(\mathrm{R})}\left(r_{i j}\right)=\left(1-r_{i j} / R_{c}\right) ; \quad w^{(\mathrm{D})}\left(r_{i j}\right)=\left[w^{(\mathrm{R})}\left(r_{i j}\right)\right]^{2} ; \quad \sigma^{2}=2 \gamma k_{\mathrm{B}} T
\end{array}
$$

Where $\sigma$ is a noise scaling prefactor, $\gamma$ is a drag scaling prefactor, and $w^{(\mathrm{R})}$ and $w^{(\mathrm{D})}$ are weighting factors for the random force fluctuations and drag force respectively. These functions are constructed in such a way that when the temperature becomes too high, the drag force increases, causing the temperature to drop. Conversely, if the temperature becomes too low, the random force increases, and the temperature rises.

\section{System Setup}

For the sake of simplicity, the solvent, polymer chains, and NP are built of the beads of the same diameter, $R_{c}=0.71 \mathrm{~nm}$, which represents the cut-off of the DPD repulsion potential. In order to prevent any preferential adhesion or repulsion between the NP and PB, the DPD intracomponent and inter-component repulsion parameters are taken equal to $a=42 k_{B} T / R_{C}$ for all the beads. This value corresponds to the parameterization of benzene obtained by matching experimental and simulated solvent compressibility. ${ }^{5}$ Excluding the channel walls, the system density is taken equal to 3 beads $/ \mathrm{R}_{\mathrm{c}}{ }^{3}$ that also corresponds to the density of liquid solvent at ambient conditions.

Simulations are performed for NPs of two radii: $R_{N P}=8 R_{c}(2.84 \mathrm{~nm})$ and $R_{N P}=8 R_{C}(5.68$ $\mathrm{nm})$. The NPs are composed of hexagonal packing lattice as illustrated in Figure S1. A set of simulations for a functionalized NP with ligand density of 0.6 ligands $/ \mathrm{nm}^{2}$ is also studied. The ligands are represented as 6-bead chains. The ligands are uniformly distributed over the NP surface bonded together in a linear fashion with $\mathrm{K}_{\mathrm{i}}=60 \mathrm{kT} / \mathrm{R}_{\mathrm{c}}{ }^{2}$ and equilibrium bond distance of $0.8 \mathrm{R}_{\mathrm{c}}$. The column wall also has the same lattice structure with a density of 12 beads $/ \mathrm{R}_{\mathrm{c}}{ }^{3}$ to prevent any penetration by the solvent.

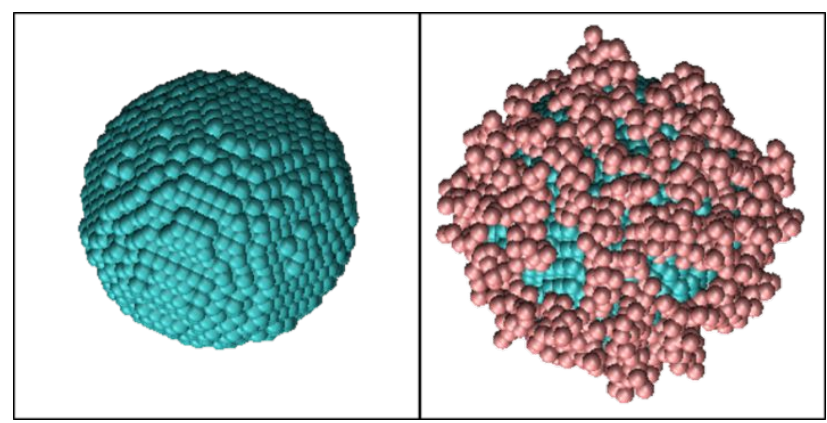

Figure S1 - Two different types of NPs studied. NP with a ligand coating of 0.6 ligands $/ \mathrm{nm}^{2}$ (left), and a bare NP (right).

The polymer brush is composed in the same manner as the ligands, with $K_{i}=60 \mathrm{kT} / \mathrm{R}_{\mathrm{c}}{ }^{2}$ and equilibrium bond distance of $0.8 \mathrm{R}_{\mathrm{c}}$. The initial PB bead of each polymer chain is frozen, $0.25 \mathrm{R}_{\mathrm{c}}$ from the surface of the wall. At initial conditions, the PB beads extend normal relative to the 
channel wall. The PB is studied under two different grafting densities: 2.4 chains $/ \mathrm{nm}^{2}$, and 0.6 chains $/ \mathrm{nm}^{2}$. These values are chosen to represent experimentally attainable grafting densities on a silica support. ${ }^{6-8}$ Adjacent PB chains are evenly spaced on the channel surface, however spacing depends on grafting density. The length of polymer chains, $n_{\text {seg }}$, ranges from 5 to 37 beads.
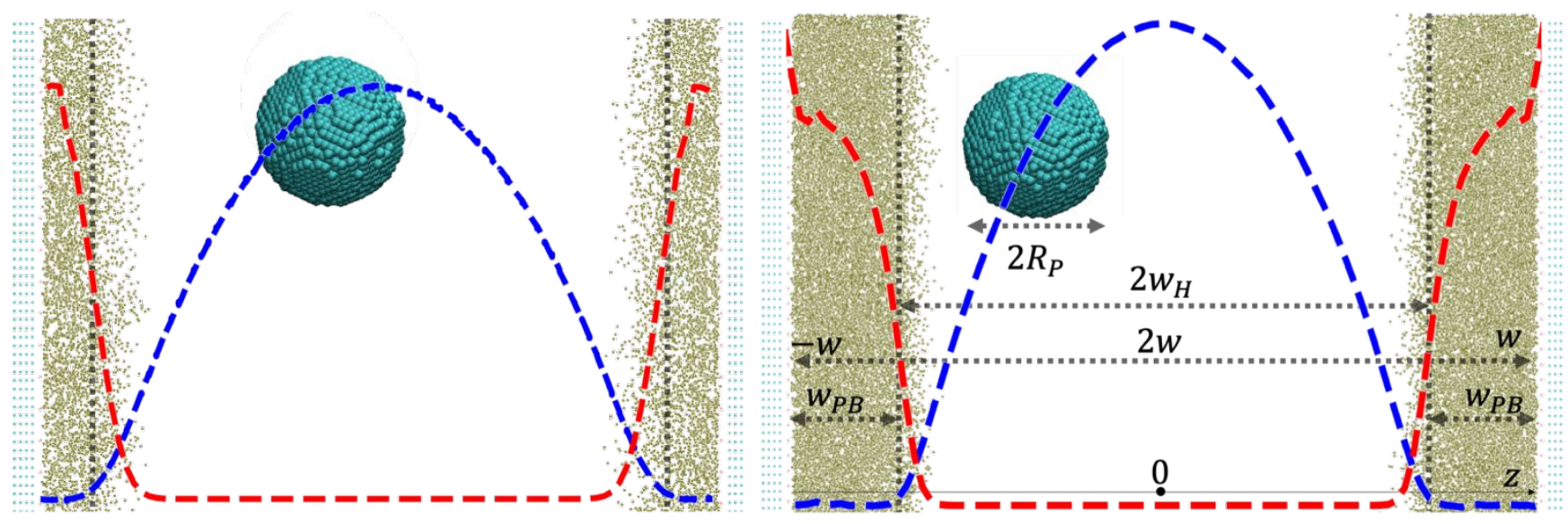

Figure S2 - Grafting densities of 0.6 chains $/ \mathrm{nm}^{2}$ (left) and 2.4 chains $/ \mathrm{nm}^{2}$ (right). Both PBs are constructed of 15 beads. Brown is the PB, blue the static channel wall, and cyan the NP. Blue dashed lines represent solvent (not shown for clarity) velocity, and red dashed line the PB density.

For $4 \mathrm{R}_{\mathrm{c}} \mathrm{NPs}$, the system is $40 \mathrm{x} 40 \mathrm{R}_{\mathrm{c}}$ in the plane of the wall, and for $8 \mathrm{R}_{\mathrm{c}}$ NPs it is $80 \mathrm{x} 80$ $\mathrm{R}_{\mathrm{c}}$. Channel width is varied to sample a wide range of aspect ratios, $\lambda=R_{N P} / r_{c}$ (from 0.1 to $0.75)$ where $r_{c}$ is the effective width of the channel. At initial state, the solvent beads are placed randomly within the boundaries of the channel wall. The PB chains are placed normal to the channel wall spaced with adjacent bead spacing within a given polymer chain equal to $0.25 R_{c}$. Distance between adjacent chains depends on grafting density as described above. The NP is placed in the center of the system. As the simulation begins, a constant force is applied to all solvent beads. This force is maintained throughout the course of the simulation. An initial equilibration run of 1 million steps with a timestep of $0.02 \tau$ is run to reach steady state, then a second 1 million step data gathering run is started. In order to ensure the entire channel is sampled, many parallel simulations are run where the initial position of the NP is changed at the beginning of the simulation. The first $10 \%$ of points is ignored to ensure the system has reached steady state with the new NP position. Distance between initial NP positions depends on channel width, however the goal is to ensure the majority of the channel is explored, which is validated through observation of the results, as illustrated in Figure S3. 

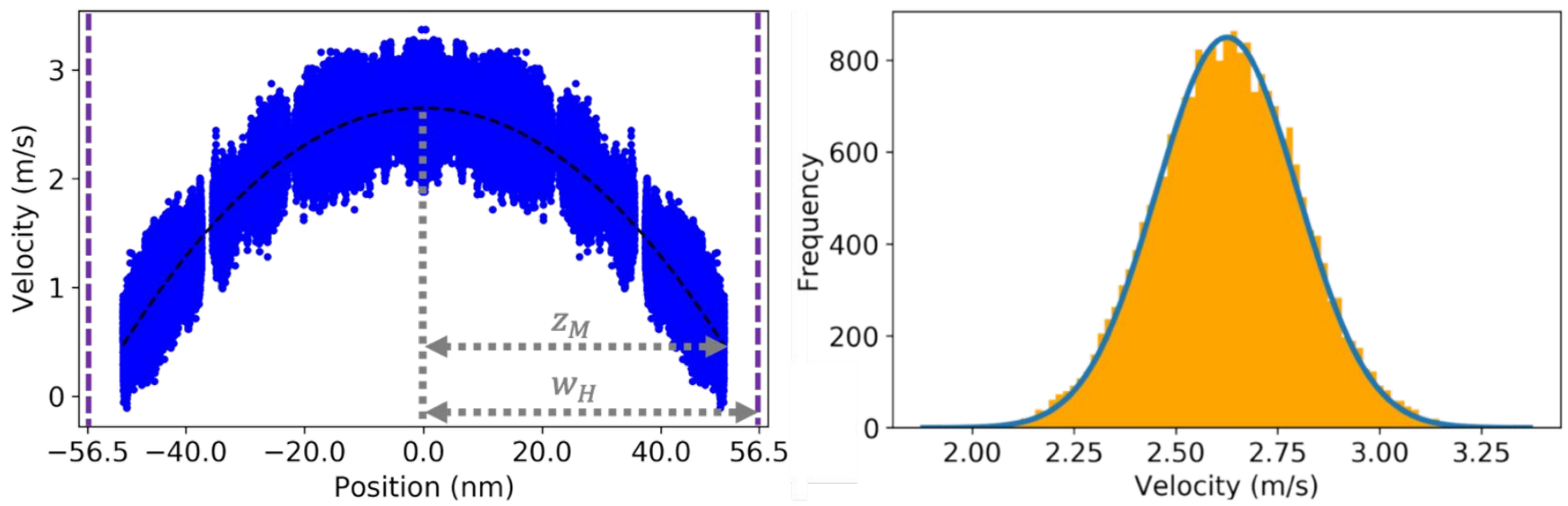

Figure S3 - Instantaneous NP velocity (blue) for the $5.68 \mathrm{~nm}$ NP in the channel of $62.7 \mathrm{~nm}$, with the PB hydrodynamic width $6.2 \mathrm{~nm}$, that corresponds to $\lambda=0.1$. Black dashed line represents the parabolic approximation for the mean velocity (left). The distribution of instantaneous NP velocities is shown for the pore center $( \pm 0.5 \mathrm{~nm})$ (right). The solid line corresponds to the expected Maxwell-Boltzmann distribution centered at the averaged NP velocity in the solvent flow.

As observed in Figure S3, the entire channel is explored. Note, because of the symmetry of the system, all data is reflected across the centerline to provide better statistics. The image provided represents 6.3 million individual data points.

\section{Excluded volume analysis}

As observed in Figure 2, the NP center of mass is excluded from the wall equal to its radius. This is an interesting result because, despite the channel thickness being defined by the average polymer brush thickness, the NP is excluded in the same manner as if it were a hard wall. However, the PB surface is soft, so that NPs fluctuate and partially penetrate beyond the mobile zone boundary $z_{M}$. To analyze the fluctuations of NP positions at the boundary of the PB, we collected statistics of the farthest distances, $z_{m}$, from the pore center achieved by the NP due to radial diffusion. The deviation of closest NP approach of a hard wall channel, $\Delta R_{H W}=w_{H}-$ $\left(z_{m}+R_{N P}\right)$ is averaged for all simulations of a certain type (bare and functionalized). In an ideal case of hard wall, $\Delta R_{H W}=0$, Figure 2, Figure $\mathrm{S} 3$.

For the bare NP, $\Delta R_{H W}=0.04 \pm 0.31 \mathrm{~nm}$, where the error is the standard deviation. The results from the simulation indicate the NP-wall exclusion behaves similarly for both soft and hard walls. Carrying out the same calculation for the functionalized NPs, we found $\Delta R_{H W}$ $=1.18 \pm 0.55 \mathrm{~nm}$. This indicates the ligands increase the effective NP radius by $1.18 \mathrm{~nm}$.

1. Hoogerbrugge, P. J.; Koelman, J. M. V. A., Simulating Microscopic Hydrodynamic Phenomena with Dissipative Particle Dynamics. Europhys Lett 1992, 19 (3), 155-160.

2. Groot, R. D.; Warren, P. B., Dissipative Particle Dynamics: Bridging the Gap Between Atomistic and Mesoscopic Simulation. J Chem Phys 1997, 107 (11), 4423-4435. 
3. Moeendarbary, E.; Ng, T. Y.; Zangeneh, M., Dissipative Particle Dynamics in Soft Matter and Polymeric Applications - a Review. Int J Appl Mech 2010, 2 (1), 161-190.

4. Moeendarbary, E.; Ng, T. Y.; Zangeneh, M., Dissipative Particle Dynamics: Introduction, Methodology and Complex Fluid Applications - a Review. Int J Appl Mech 2009, 1 (4), 737-763. 5. Cheng, J. L.; Vishnyakov, A.; Neimark, A. V., Morphological Transformations in Polymer Brushes in Binary Mixtures: DPD Study. Langmuir 2014, 30 (43), 12932-12940.

6. Berendsen, G. E.; Galan, L. D., Geometrical Model for Chemically Bonded Tms and Pds Phases. J Liq Chromatogr 1978, 1 (4), 403-426.

7. Pous-Torres, S.; Torres-Lapasio, J. R.; Garcia-Alvarez-Coque, M. C., Comparison of the Performance of Chromolith Performance RP-18e, 1.8-mu m Zorbax Eclipse XDB-C18 and XTerra MS C18, Based on Modelling Approaches. Anal Bioanal Chem 2013, 405 (7), 22192231.

8. Cabrera, K.; Machtejevas, E., New Generation Monolithic Silica Columns for Fast, High Resolution Drug Separations without High Pressures. Lc Gc N Am 2012, 37-37. 